\title{
Dark Matter Problem in the Local Supercluster
}

\author{
Dmitry Makarov ${ }^{1}$ and Igor Karachentsev ${ }^{1}$ \\ ${ }^{1}$ Special Astrophysical Observatory of the Russian Academy of Sciences, Nizhnij Arkhyz, \\ Zelenchukskij region, Karachai-Cirkassian Republic, Russia 369167 \\ email: dim@sao.ru
}

\begin{abstract}
The Local Supercluster is an ideal laboratory to study distribution of luminous and dark matter in the nearby Universe. The 1100 small groups have been selected using algorithm based on assumption that a total energy of physical pair of galaxies must be negative. The properties of the groups have been considered.
\end{abstract}

Keywords. dark matter

\section{Algorithm}

We used an selection algorithm based on assumption that a total energy of physical pair of galaxies must be negative (Makarov \& Karachentsev 2000). Individual mass of the galaxies are estimated from their K-luminosity using appropriate value of M/L. It is chosen to reproduce the well known nearby groups of galaxies, like the Centaurus A, M 81, M 83 and IC 342.

\section{Parameters of the groups}

The 5687 galaxies of 10571 (about 54\%) with $V_{\mathrm{LG}}<3500$ and $|b|>15^{\circ}$ have been gathered in 1127 groups. The median velocity dispersion in groups with $\geqslant 5$ members is $88 \mathrm{~km} \mathrm{~s}^{-1}$, and the median harmonic radius is $222 \mathrm{kpc}$. We find the median mass-to-Kluminosity ratio for the groups to be 26 in solar units using a projected mass estimator (Heisler et al. 1985). That shows presence of moderate amount of dark matter in the considered groups.
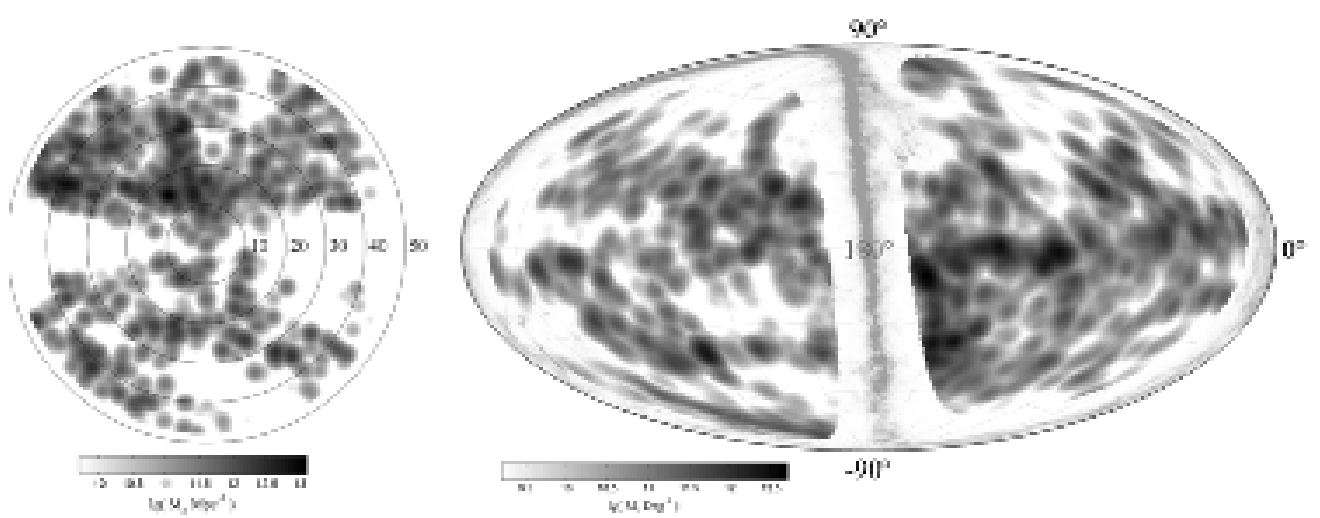

Figure 1. The mass distribution in the Local Supercluster. The left panel presents distribution of surface mass density in the Local Supercluster plane. The right panel shows an all sky map of surface mass density in the Supergalactic coordinates. 


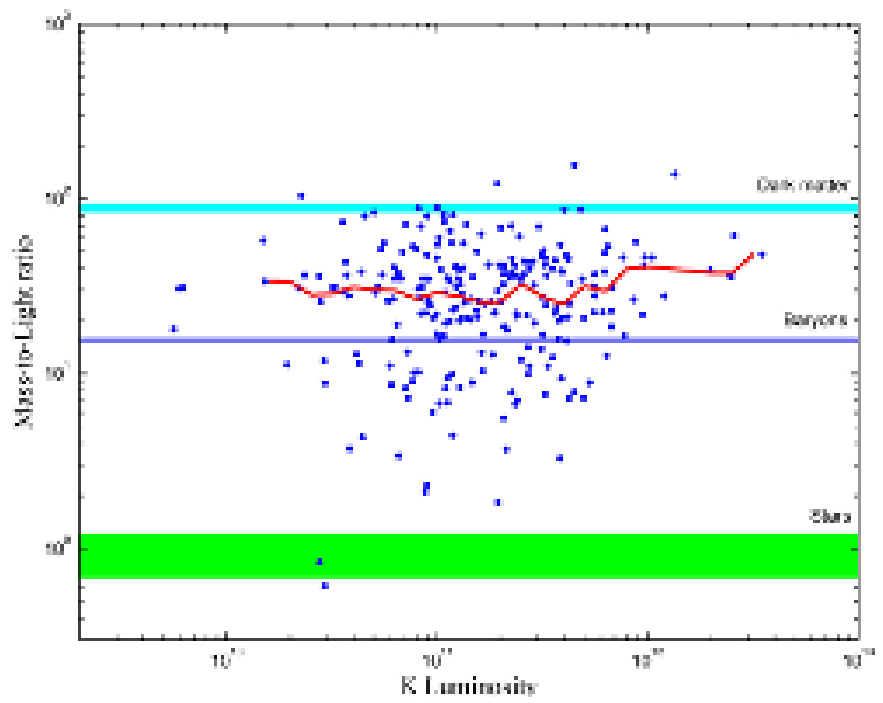

Figure 2. The mass-to-light ratio versus K-band luminosity. The dots show the individual groups. The winding line represents the running median of $\mathrm{M} / \mathrm{L}$. The horizontal lines show the mean value for stars $M / L=0.95 \pm 0.27$ (Bell et al. 2003), expected M/L ratio for baryonic $\Omega_{b} h^{2}=0.02229$ and dark $\Omega_{m} h^{2}=0.1277$ matter in standard cosmology (Spergel et al. 2007).

\section{Dark matter in the Local Supercluster}

Based on our algorithm for group selection we derived the median mass-to-light ratio of groups $M / L_{K} \sim 26$ that was used to trace the distribution of mass via light distribution in the Local Supercluster (LSC). The mean K-band luminosity density within the considered volume is $j_{K}=3.96 \times 10^{8} \mathrm{~L}_{\odot} \mathrm{Mpc}^{-3}$, being in good agreement with the global luminosity density $4.19 \pm 0.63 \times 10^{8} \mathrm{~L}_{\odot} \mathrm{Mpc}^{-3}$ from $2 \mathrm{dF}$ survey (Cole et al. 2001) and $4.24 \times 10^{8} \mathrm{~L}_{\odot} \mathrm{Mpc}^{-3}$ from 2MASS and SDSS surveys (Bell et al. 2003). Thus, the mean total mass density inside the LSC volume is $1.0 \times 10^{10} \mathrm{M}_{\odot} \mathrm{Mpc}^{-3}$ that corresponds to $\Omega_{m}=0.07$. This value amounts only a quarter of the global density $\Omega_{m}=0.27$ in standard cosmology (Spergel et al. 2007). Even the total mass-to-light ratio of the Virgo cluster $\left(M / L_{K} \sim 62\right)$ is not enough to reach the global value $M / L_{K} \sim 90$ expected for $\Omega_{m}=0.27$. To adjust the ensemble averaged value $\Omega_{m}=0.07$ with the global density one needs to suppose that a significant part of the dark matter on the LSC scale is not associated with system of galaxies.

\section{Acknowledgements}

This work was supported by DFG-RFBR grant 06-02-04017 and RFBR grant 07-0200005 .

\section{References}

Bell et al. 2003, ApJ 149, 289

Cole et al. 2001, MNRAS 326, 255

Heisler et al. 1985, ApJ 298, 8

Makarov \& Karachentsev, 2000, ASPC 209, 40

Spergel et al. 2007, ApJS 170, 377 